\title{
Atuação dos cirurgiões-dentistas na capacitação e desenvolvimento de ações de saúde bucal pelo agente comunitário
}

\author{
Dentist surgeons 'activities in the training and development of healthcare actions \\ by the community health agent \\ Actuación de los odontólogos en la capacitación y desarrollo de acciones de salud bucal \\ por el agente comunitario
}

\author{
André Luiz Marçal TERRERI \\ Mariângela Monteiro de Melo BALTAZAR \\ Marina BERTI \\ Danielle PORTINHO \\ Daniela Pereira LIMA
}

Curso de Odontologia. Universidade Estadual do Oeste do Paraná/UNIOESTE, Cascavel/PR, Brasil

\begin{abstract}
Resumo
O presente estudo buscou analisar a percepção e atuação dos cirurgiões-dentistas inseridos na estratégia saúde da família do Município de Cascavel-PR, acerca da capacitação e desenvolvimento de ações de vigilância à saúde bucal pelo agente comunitário de saúde. A coleta dos dados foi realizada em todas as unidades de saúde da família que possuíam equipes de saúde bucal sendo utilizado um questionário estruturado, autoaplicável, composto por questões especialmente elaboradas para a pesquisa. Após a categorização, os dados coletados foram digitados em planilha eletrônica criada no software Epi Info, versão 3.5.1. O Teste Exato de Fisher com nível de significância de 5\% foi utilizado para verificação de associação entre variáveis. Os resultados demonstraram pouca articulação entre cirurgiões-dentistas e agente comunitário de saúde na capacitação e desenvolvimento de ações de saúde bucal. Ter o titulo de especialista em odontologia em saúde coletiva não foi um fator significativo no treinamento e delegação de ações de saúde bucal. Mudanças no modelo de prestação dos serviços de saúde no município vão exigir a existência de um programa de educação permanente, que possibilite renovar e adquirir os conhecimentos de saúde bucal, para que os Agentes Comunitários de Saúde consigam atuar como atores na vigilância em saúde bucal.
\end{abstract}

Descritores: Saúde Bucal; Agentes Comunitários de Saúde; Capacitação em Serviço.

\section{Abstract}

The present study sought to analyze the perception and performance of dental surgeons inserted in the family health strategy of the Municipality of Cascavel-PR, about the training and development of oral health surveillance actions by the community health agent. The data collection was performed in all the family health units that had oral health teams and a structured, self-administered questionnaire was used, composed by specially elaborated questions for the research. After the categorization, the collected data were typed in a spreadsheet created in Epi Info software, version 3.5.1. Fisher's exact test with significance level of 5\% was used to verify association between variables. The results showed little articulation between dental surgeons and community health agent in the training and development of oral health actions. Having the title of specialist in public health dentistry was not a significant factor in the empowerment and delegation of oral health actions. Changes in the model of provision of health services in the municipality will require the existence of a permanent education program that will make it possible to renew and acquire oral health knowledge so that Community Health Agents can act as actors in oral health surveillance.

Descriptors: Oral Health; Community Health Workers; Inservice Training.

\section{Resumen}

El presente estudio buscó analizar la percepción y actuación de los odontólogos insertados en la estrategia salud de la familia del Municipio de Cascavel-PR, acerca de la capacitación y desarrollo de acciones de vigilancia a la salud bucal por el agente comunitario de salud. La recolección de los datos fue realizada en todas las unidades de salud de la familia que poseían equipos de salud bucal siendo utilizado un cuestionario estructurado, auto-aplicable, compuesto por cuestiones especialmente elaboradas para la investigación. Después de la categorización, los datos recolectados fueron digitados en una hoja de cálculo creada en el software Epi Info, versión 3.5.1. La prueba exacta de Fisher con un nivel de significancia del $5 \%$ se utilizó para la verificación de asociación entre variables. Los resultados demostraron poca articulación entre cirujanos-dentistas y agente comunitario de salud en la capacitación y desarrollo de acciones de salud bucal. Tener el título de especialista en odontología en salud colectiva no fue un factor significativo en el entrenamiento y delegación de acciones de salud bucal. Los cambios en el modelo de prestación de los servicios de salud en el municipio van a exigir la existencia de un programa de educación permanente, que posibilite renovar y adquirir los conocimientos de salud bucal, para que los Agentes Comunitarios de Salud consigan actuar como actores en la vigilancia en salud bucal.

Descriptores: Salud Bucal; Agentes Comunitarios de Salud; Capacitación en Servicio.

\section{INTRODUÇÃO}

$\mathrm{Na}$ tentativa de redesenhar o modelo de atenção e promover a qualidade dos serviços de saúde bucal na atenção básica, surge a possibilidade do trabalho multiprofissional na Estratégia Saúde da Família (ESF). Neste contexto, um profissional assume um papel de destaque - o agente comunitário de saúde (ACS). O ACS tem um papel fundamental no desenvolvimento de relações de vínculo e responsabilização entre as equipes e a população.

Teoricamente, é a garantia da continuidade das ações de saúde e a longitudinalidade do cuidado, pois é o elemento da equipe que, pela própria natureza do trabalho, percorre os domicílios podendo identificar problemas de saúde ${ }^{1}$ e condições de riscos ligados à ocupação, renda e escolaridade, que influenciam na distribuição de cárie ${ }^{2}$, doença periodontal $^{3}$, perda dentaria ${ }^{4}$ e edentulismo ${ }^{5}$. Na restruturação do modelo de saúde bucal na ESF é importante a compreensão de que entre as atribuições básicas dos ACS estão as que fazem parte do conhecimento da área, as que fazem parte do acompanhamento, monitoramento, prevenção de 
doenças e promoção da saúde e as ações educativas, incluindo as relacionadas à saúde bucal ${ }^{6}$. Nesse sentido, para ampliar o aproveitamento das forças trabalhadoras da equipe, principalmente dos ACS, é importante a inserção de conteúdos relacionados à saúde bucal na sua capacitação, para que atuem no sentido de mudanças dos hábitos da família e na busca do autocuidado com eficiência. O ACS conhece a intimidade, moradia, modo de vida das pessoas da área em que atua, contribuindo, desse modo, para a definição do tipo de assistência necessária, sendo um elo entre as necessidades de saúde das pessoas e o que pode ser feito para a melhoria das condições de vida da comunidade ${ }^{7}$. O ACS em articulação com a ESB e devidamente capacitado pode contribuir para fortalecer $O$ conhecimento da população no enfrentamento dos problemas de saúde, além de auxiliar a equipe de saúde bucal na identificação das famílias mais vulneráveis que necessitam de ações específicas, bem como, melhorar o acesso e a utilização dos serviços primários de saúde, a fim de evitar a assistência odontológica tardia, reduzindo a necessidade de consultas de urgência ${ }^{8}$. Entretanto, uma revisão ${ }^{9}$ das publicações no período de 2001 a 2008, sobre a atuação da equipe de saúde bucal (ESB) na estratégia saúde da família (ESF), mostrou que, na grande maioria dos municípios pesquisados, as ações da ESB na ESF são calcadas no modelo tradicional de atenção, indicando que o incentivo financeiro parece não ser suficiente para promover as mudanças desejadas. Executar ações de saúde bucal fora dos limites físicos da unidade de saúde (US), por outros profissionais, representa ao mesmo tempo um avanço significativo e um grande desafio, já que historicamente a odontologia construiu suas práticas dependentes da clínica e centradas na figura do cirurgião-dentista (CD). Assim, este estudo foi delineado com o objetivo de analisar a percepção e atuação dos CDs inseridos na ESF, do Município de Cascavel-PR, acerca da capacitação e desenvolvimento de ações de vigilância à saúde bucal (promoção e prevenção) pelo ACS, ao entendê-los como integrantes essenciais da equipe de saúde.

\section{MATERIAL E MÉTODO}

Trata-se de estudo censitário, transversal, com abordagem quantitativa, no qual a população do estudo foi constituída pelos CDs de todas as US com ESB do município de Cascavel-PR. Para a coleta dos dados foi utilizado questionário estruturado, autoaplicável, composto por questões especialmente elaboradas para a pesquisa. Participaram da amostra todos os CDs que concordaram em participar da pesquisa e assinaram o termo de consentimento livre esclarecido (TCLE). Foram excluídos da amostra todos os profissionais que não concordaram e/ou não assinaram TCLE ou não estavam presentes por motivo de licença no período da coleta dos dados.

A análise e discussão dos dados coletados se pautaram na necessidade de incluir as atividades de vigilância em saúde bucal na rotina do trabalho dos ACS e a importância do CD orientar o seu trabalho. O município está localizado na região oeste do Estado do Paraná, com população estimada de 319.618 habitantes, com esperança de vida de 75,7 anos e renda per capita em reais de 1003,38, situação que o classifica em $4^{\circ}$ melhor município da Unidade da Federação e $113^{\circ}$ na classificação nacional com relação ao Índice de Desenvolvimento Humano $(0,782)^{10}$.

Inicialmente foi consultado o Secretário de Saúde do Município para informá-lo a respeito do objetivo do estudo e do posterior uso dos dados coletados, a fim de obter apoio para a realização do estudo. Desde então, o projeto de pesquisa foi conduzido dentro dos padrões exigidos pela Resolução 196/CNS e aprovado pelo Comitê de Ética em Pesquisa em Seres Humanos da Universidade Estadual do Oeste do Paraná - UNIOESTE, com Certificado de Apresentação para Apreciação Ética número: 64461317.3.0000.0107. Os CDs aceitaram participar do estudo e foram informados em relação à finalidade e ao sigilo das informações coletadas e assinaram o Termo de Consentimento Livre e Esclarecido.

Após a coleta dos dados, os questionários foram digitados em planilha eletrônica criada no software Epi Info, versão 3.5.1. Para a análise estatística, os dados referentes ao tempo de graduação e atuação na ESF foram reagrupados em dois grupos: Menos de dez anos ( 1 a 5 anos e 6 a 10 anos) Mais de dez anos (11 a 15 anos e 16 ou mais). $\mathrm{O}$ envolvimento da equipe de saúde bucal com o restante da equipe de saúde da família foi reagrupado da seguinte forma: Baixo (Muito baixo e Baixo), médio e alto (Alto e muito alto). As atividades de saúde bucal que o ACS realiza durante as visitas também foram reagrupados para fins estatísticos em: Não (Nenhuma atividade desenvolvida) e Sim (Educação em saúde bucal, escovação supervisionada, evidenciação do biofilme, aplicação de fluoretos e exame bucal para identificação de usuários com necessidade de encaminhamento). Foi realizada a estatística descritiva dos dados, constituindo-se as frequências para cada uma das variáveis do estudo, calculando-se as medidas estatísticas apropriadas à natureza de cada uma delas e fazendo-se a apresentação tabular desses resultados. Para a verificação da associação entre as variáveis: Especialização em saúde coletiva, Capacitação aos ACS e Implementação de atividades em saúde bucal foi utilizado o teste Exato de Fisher com nível de significância de 5\%. 


\section{RESULTADOS}

A coordenação municipal de saúde bucal informou em junho de 2017, período em que foi realizada a pesquisa, que havia possuía 20 unidades de saúde com Equipes de Saúde Bucal, sendo que, em 19 unidades os dentistas foram entrevistados. Em apenas uma US o CD não participou da pesquisa devido afastamento médico. As equipes de saúde bucal do Município de Cascavel são compostas na sua grande maioria $(68,4 \%)$ por $\mathrm{CD}$ pertencentes ao sexo feminino. A maioria $(63,2 \%)$ dos entrevistados possui acima de 11 anos de formados, entretanto, $89,6 \%$ dos entrevistados disseram estar inseridos na ESF a menos de 10 anos. $O$ total de profissionais com título de especialista trabalhando na estratégia de saúde da família foi de $68,4 \%$, podendo observar uma tendência nítida em optar pela especialidade em "saúde coletiva e da família" (61,5\%), aspecto importante para aperfeiçoamento da odontologia na atenção básica à saúde (Quadro 1).

Quadro 1. Distribuição numérica e percentual das especialidades concluídas dos cirurgiões dentistas inseridos na ESF. CascavelPR, Brasil, 2017.

\begin{tabular}{|l|c|c|}
\hline \multicolumn{1}{|c|}{ Especialidade } & n & \% \\
\hline $\begin{array}{l}\text { Cirurgia Traumatologia } \\
\text { Bucomaxilofacial }\end{array}$ & 02 & 13,3 \\
\hline Dentística & 0 & 0 \\
\hline $\begin{array}{l}\text { Disfunção Temporomandibular e Dor } \\
\text { Orofacial }\end{array}$ & 0 & 0 \\
\hline Endodontia & 0 & 0 \\
\hline Estomatologia & 0 & 0 \\
\hline Radiologia Odontológica e Imaginologia & 01 & 7,6 \\
\hline Implantodontia & 02 & 13,3 \\
\hline Odontologia Legal com & 01 & 7,6 \\
\hline Odontologia do Trabalho & 0 & 0 \\
\hline $\begin{array}{l}\text { Odontologia para Pacientes } \\
\text { Necessidades Especiais }\end{array}$ & 0 & 0 \\
\hline Odontopediatria & 05 & 0 \\
\hline Ortodontia & 0 & 0 \\
\hline Ortopedia Funcional dos Maxilares & 0 & 0 \\
\hline Patologia Bucal & 01 & 7,6 \\
\hline Periodontia & 0 & 0 \\
\hline Prótese Buco-Maxilo-Facial & 01 & 7,6 \\
\hline Prótese Dentária & 08 & 61,5 \\
\hline Saúde Coletiva e da Família &
\end{tabular}

Poucos profissionais $(15,8 \%)$ consideraram baixo o envolvimento da ESB com o restante da ESF, sendo que, a periodicidade com que acontece reunião entre toda a equipe da unidade de saúde, incluído a ESB, foi $100 \%$ para semanal/quinzenal. Durante o exercício da profissão como CD na ESF, $63,2 \%$ dos entrevistados ofereceram capacitação sobre saúde bucal para os agentes comunitários, sendo a maioria $(52,6 \%)$, com periodicidade anual (Tabela 1$)$.

Dentre os que ofereceram capacitação, os conteúdos mais tratados foram a doença cárie em primeiro, seguidos de periodontite e câncer de boca, ficando a gengivite em terceiro. Traumatismo dentário e edentulismo também fizeram parte do conteúdo de capacitação realizada, respectivamente, por dois e três cirurgiões-dentistas. O tema fluorose dentária nunca foi objeto de capacitação para os ACS. Pode-se observar ainda na Tabela 1 que $73,7 \%$ dos dentistas afirmaram que nenhuma atividade de saúde bucal, tais como educação em saúde bucal e escovação supervisionada são desenvolvidas durante as visitas domiciliares realizadas pelos ACS.

Tabela 1. Distribuição numérica e percentual sobre capacitação e desenvolvimento de ações de saúde bucal pelo ACS na ESF Cascavel- PR, Brasil, 2017.

\begin{tabular}{|c|c|c|}
\hline Variável & $\mathbf{n}$ & $\%$ \\
\hline $\begin{array}{l}\text { Ofereceu capacitação sobre } \\
\text { saúde bucal aos ACS } \\
\text { Sim } \\
\text { Não }\end{array}$ & $\begin{array}{l}12 \\
07\end{array}$ & $\begin{array}{l}63,2 \\
36,8\end{array}$ \\
\hline $\begin{array}{l}\text { Frequência com que oferece } \\
\text { capacitação para os ACS } \\
\text { Anualmente } \\
\text { Mensalmente } \\
\text { Semanalmente } \\
\text { Diariamente } \\
\text { Nunca }\end{array}$ & $\begin{array}{l}10 \\
01 \\
00 \\
01 \\
07\end{array}$ & $\begin{array}{c}52,6 \\
5,3 \\
00 \\
5,3 \\
36,8\end{array}$ \\
\hline $\begin{array}{l}\text { Em sua opinião, sinais } \\
\text { individuais de risco em saúde } \\
\text { bucal podem ser identificados } \\
\text { pelos ACS } \\
\text { Sim } \\
\text { Não }\end{array}$ & $\begin{array}{l}19 \\
00\end{array}$ & $\begin{array}{c}100 \\
00\end{array}$ \\
\hline $\begin{array}{l}\text { ACS realizam atividades de } \\
\text { saúde bucal durante as visitas } \\
\text { domiciliares } \\
\text { Sim } \\
\text { Não }\end{array}$ & $\begin{array}{l}05 \\
14\end{array}$ & $\begin{array}{l}26,3 \\
73,7\end{array}$ \\
\hline $\begin{array}{l}\text { Com qual frequência os ACS } \\
\text { desenvolvem atividades } \\
\text { preventivas, em espações sociais } \\
\text { na área de abrangência da } \\
\text { Unidade de Saúde }\end{array}$ & & \\
\hline $\begin{array}{l}\text { Anualmente } \\
\text { Mensalmente }\end{array}$ & $\begin{array}{l}00 \\
00\end{array}$ & $\begin{array}{l}00 \\
00\end{array}$ \\
\hline Semanalmente & 01 & 5,2 \\
\hline $\begin{array}{l}\begin{array}{l}\text { Somente em } \\
\text { específicas }\end{array} \\
\text { campanhas }\end{array}$ & 03 & 15,8 \\
\hline Nunca & 15 & 79,0 \\
\hline Total & 19 & 100 \\
\hline
\end{tabular}

Resultado semelhante também foi encontrado para outros espaços sociais na área de abrangência da unidade de saúde, como escolas e creches, onde 79\% dos entrevistados relataram que os ACS não realizam atividades de saúde bucal (Tabela 1). Quando questionados sobre a delegação de função na identificação dos sinais de risco pelo ACS durante suas visitas domiciliares $100 \%$ dos entrevistados concordam com essa ação, sendo que, na opinião dos entrevistados, os sinais de risco em saúde bucal que poderiam ser identificados pelos ACS estavam a necessidade de prótese e a falta de acesso a escova e dentifrício fluoretado e Limitações sociais, estéticas e funcionais ligadas a problemas bucais (Quadro 2).

A tabela 2 apresenta os resultados dos testes de associação entras as variáveis do estudo. Foi 
possível observar que ser especialista na área de saúde coletiva e da família não proporcionou aumentou dos ACS capacitados e tampouco o desenvolvimento de ações de saúde bucal na rotina deste profissional.

Quadro 2. Distribuição numérica e percentual dos sinais individuais de risco em saúde bucal que podem ser identificadas pelos ACS, na opinião do cirurgião dentista inserido na ESF. Cascavel- PR, Brasil, 2017.

\begin{tabular}{|l|c|c|}
\hline Sinais individuais de risco em saúde bucal & n & $\%$ \\
\hline Cavidades nos dentes & 07 & 37,0 \\
\hline Falta de escovação diária & 09 & 47,3 \\
\hline $\begin{array}{l}\text { Falta de acesso à escova e dentifrício } \\
\text { fluoretado }\end{array}$ & 13 & 68,4 \\
\hline $\begin{array}{l}\text { Exposição excessiva ao dentifrício } \\
\text { fluoretado }\end{array}$ & 05 & 26,3 \\
\hline Sangramento gengival & 04 & 21,3 \\
\hline Mobilidade dentária & 04 & 21,3 \\
\hline $\begin{array}{l}\text { Lesão de tecidos moles ou sintomas ligados } \\
\text { à possibilidade de câncer bucal }\end{array}$ & 05 & 26,3 \\
\hline Necessidade de prótese & 14 & 73,7 \\
\hline $\begin{array}{l}\text { Limitações sociais, estéticas e funcionais } \\
\text { ligadas a problemas bucais }\end{array}$ & 13 & 68,4 \\
\hline Outros & 00 & 00 \\
\hline
\end{tabular}

Tabela 2- Associação entre as variáveis: Especialização em saúde coletiva, Capacitação aos ACS e Desenvolvimento de atividades em saúde bucal. Cascavel- PR, Brasil, 2017.

\begin{tabular}{cccc}
\hline Variáveis & $\begin{array}{c}\text { Teste de } \\
\text { Associação }\end{array}$ & $\begin{array}{c}\text { p- } \\
\text { valor }\end{array}$ & Significância* \\
\hline $\begin{array}{c}\text { Especialização em } \\
\text { saúde coletiva } \\
\text { X }\end{array}$ & $\begin{array}{c}\text { Teste } \\
\text { Exato de } \\
\text { Fisher }\end{array}$ & 0,2966 & NS \\
$\begin{array}{c}\text { Capacitação aos } \\
\text { ACS }\end{array}$ & $\begin{array}{c} \\
\text { Teste }\end{array}$ & 0,6641 & NS \\
$\begin{array}{c}\text { Especialização em } \\
\text { saúde coletiva } \\
X\end{array}$ & $\begin{array}{c}\text { Exato de } \\
\text { Fisher }\end{array}$ & & \\
$\begin{array}{c}\text { Desenvolvimento de } \\
\text { atividades em saúde } \\
\text { bucal pelo ACS }\end{array}$ & & & \\
* NS= Não significante & & & \\
\end{tabular}

\section{DISCUSSÃO}

O presente trabalho teve o intuito de analisar a percepção e atuação dos CDs no que concernem as ações de saúde bucal que poderiam estar inseridas nas práticas dos ACS. Embora a Odontologia se mostre desenvolvida clinicamente e seja fundamental na Atenção Básica $(\mathrm{AB})$, sua prática clínica isolada não consegue responder em níveis significativos às demandas dos problemas de saúde bucal da comunidade. Há necessidade de reforçar ações de vigilância à saúde bucal (prevenção e promoção) que contribuam na redução das iniquidades e exclusão em saúde e melhoria do quadro epidemiológico de saúde bucal. Para os CDs que trabalham no sistema único de saúde (SUS) a busca pelo aperfeiçoamento, principalmente por meio dos cursos de pós-graduação em saúde coletiva e da família certamente venha contribuir na complementação da formação de profissionais que sejam capazes de prestar atenção integral mais humanizada, trabalhar em equipe e compreender melhor a realidade em que vive a população. No estudo em questão, entre os profissionais que possuíam pós-graduação a especialidade em saúde coletiva e da família foi a mais frequente, diferentemente quando analisamos a distribuição nacional por especialidade no Brasil ${ }^{11}$, que entre as dez especialidades mais frequentes a área de saúde coletiva e da família ocupa a décima posição e no estado do Paraná $^{12}$ a décima primeira.

Neste estudo foi possível observar que profissionais com especialidade em saúde coletiva e da família, quando comparado aos CDs que não possuíam ou possuíam em outra área, não proporcionou estatisticamente, maior ocorrência de capacitação ou desenvolvimento de ações de saúde bucal por parte dos ACS durante suas visitas domiciliares. Os agentes capacitados para orientar as famílias sobre questões de saúde bucal poderiam aumentar de forma significativa a cobertura de ações de vigilância à saúde bucal, quando comparado à ESB trabalhando de forma isolada. Uma ESB, geralmente é composta por dois profissionais, no máximo três, enquanto que o número de ACS é definido de acordo com base populacional, critérios demográficos, epidemiológicos e socioeconômicos ${ }^{1}$, sendo que estes, pela própria natureza do seu trabalho, realizam visitas domiciliares com muito mais frequência que a ESB. Reuniões entre toda equipe da US, bem como sua frequência, são momentos importantes para sensibilizar que a saúde bucal deve ser entendida como objeto de intervenção de todos os profissionais da equipe e não exclusivamente dos que trabalham na área odontológica.

No estudo pode se verificar que a frequência de reuniões entre os profissionais da saúde em todas as 19 unidades foi semanal ou quinzenal, um aspecto bastante positivo para socializar propostas de ações em saúde bucal. Apesar da assiduidade das reuniões, nunca houve capacitação em saúde bucal para os ACS em $36,8 \%$ das US e na maioria $(52,6 \%)$ das que ocorreram a periodicidade foi anual, o que permite subentender que o planejamento de ações de vigilância à saúde bucal no município também pouco avançou para serem desenvolvidas. Este fato fica evidente quando verificamos que em $73,6 \%$ das US os ACS pertencentes a elas não realizam nenhuma atividade de saúde bucal durante suas visitas domiciliares, aspecto que inviabiliza uma maior cobertura populacional e percepção da comunidade sobre saúde bucal. Quando os agentes comunitários estão capacitados para exercerem essas atividades, pode haver o aumento da consciência da população para o autocuidado em saúde bucal. Esse fato é corroborado pelo estudo ${ }^{8}$ realizado no interior de São Paulo, o qual foi possível verificara mudanças significativas nos conhecimentos de saúde bucal por 
parte de mulheres e de mães da área de atuação dos agentes, que foram capacitados a realizar educação em saúde junto à comunidade. A capacitação em saúde bucal dos ACS em Alagoinhas/BA possibilitou a aquisição de conhecimento técnico e reforçou sua capacidade de ser um agente cuidador permitindo que pudesse desenvolver escovação supervisionada, palestra e aplicação de flúor, bem como, possuía forte influência na marcação da consulta odontológica. $\mathrm{O}$ estudo destacou ainda que o ACS em seu processo de trabalho influenciou as ações internas e externas da USF, tornando-se determinante para a articulação das equipes de saúde bucal com a comunidade ${ }^{13}$.

Apesar da existência de experiências exitosas quanto à capacitação e desenvolvimento de ações de saúde bucal por ACS, há diversas pesquisas ${ }^{14-19}$ que avaliaram os conhecimentos sobre saúde bucal pelos ACS e demonstraram lacunas de conhecimentos e necessidade de capacitação para que atuem como profissionais capazes exercer vigilância à saúde bucal. A saúde bucal, parte integrante da saúde geral dos indivíduos, configura-se de primordial relevância para que os agentes de saúde dominem seus conhecimentos básicos visando à promoção, prevenção e recuperação na microárea de atuação ${ }^{19}$. Planejar, gerenciar e avaliar as ações desenvolvidas pelos ACS em conjunto com os outros membros da equipe passa ser para o cirurgião-dentista um desafio, inclusive pela sua própria formação acadêmica biomédica com foco no trabalho individual $\mathrm{e}$ exercício privado da profissão, quadro inadequado ao SUS/ESF ${ }^{20,21}$. Há necessidade de introduzir novas práticas na rotina dos ACS para que o modelo de atenção à saúde bucal vá além da consulta odontológica na UBS e ações esporádicas sem continuidade e a devida longitudinalidade do cuidado. Requer transformar um sistema de atenção à saúde essencialmente fragmentado, reativo e episódico que se organiza comumente para as agudizações de condições crônicas, focado no atendimento clínico da doença, para outro sistema que seja mais proativo, integrado e contínuo ${ }^{1}$, focado nas pessoas e nas famílias e voltados para a prevenção e manutenção da saúde bucal.

Devemos considerar que maioria das doenças e agravos em saúde bucal pode ser classificada como condições crônicas, que iniciam e evoluem lentamente, usualmente ocasionadas por múltiplas causas e que podem ser enfrentadas numa lógica de intervenção preventiva e de vigilância sobre os fatores que a ocasionam. Sob essa ótica, os autores consideram que o ciclo de capacitação dos ACS deva habilitá-lo para que possam identificar sinais de risco em saúde bucal, importantes para o planejamento de ações de vigilância à saúde (falta de acesso à escova e dentifrício fluoretado, exposição excessiva ao dentifrício fluoretado, sangramento gengival) no contexto familiar, bem como, na organização da demanda para o tratamento curativo (cavidade nos dentes, mobilidade dentária, lesões dos tecidos moles, necessidade de prótese e limitações sociais, estéticas e funcionais ligadas a problemas bucais). Kusma et $\mathrm{al}^{22}$ propõem um modelo teórico para avalição das práticas de promoção da saúde bucal que pode ser empregado no planejamento das ações de saúde bucal pelo ACS e que tem como pilares a equidade, a participação e a sustentabilidade. Dessa forma, a equidade deve considerar as especificidades dos grupos populacionais, em que as necessidades das pessoas orientam a distribuição das oportunidades e de bem estar.

A participação é um processo no qual diferentes grupos participam na identificação das necessidades ou dos problemas de saúde e atuam como corresponsáveis no planejamento e execução de soluções adequadas. Já a sustentabilidade envolve uma análise da ação para que seja economicamente viável (recursos), socialmente justa (equidade, vulnerabilidade), culturalmente aceita (diversidade, participação) e psicologicamente saudável (autonomia, empoderamento). É preciso lidar com o fato da Odontologia, geralmente, não estar presente desde o início do programa, porém, isto não acarretar prejuízos no processo de integralização dos profissionais correlacionados. O estabelecimento de incentivo para financiamento de ações e da inserção de profissionais de saúde bucal na $\mathrm{ESF}^{23}$ foi criado há 17 anos e no município de que se trata, $85 \%$ das ESB estão inseridas há menos de 10 anos $(50 \%$ até 5 anos e 35\% de 6 a 10 anos). Apesar do curto período de implantação desta proposta de reorganização da saúde bucal, a capacitação para o ACS no município pouco avançou para que este profissional pudesse contribuir nas ações de vigilância à saúde e organização da demanda. Há indicações de que os CD podem desempenhar papel importante na organização das práticas, entretanto, quando sustentados por uma razoável gestão e organização dos serviços de saúde bucal, em que se encontram inseridos $^{24}$. Significa desenvolver ações, articular instituições e promover acesso aos trabalhadores, para formação e garantia de educação permanente e continuada aos profissionais de saúde das equipes de saúde da família ${ }^{1}$.

Neste contexto, uma importante função da universidade é a de identificar corretamente os problemas de saúde de cada município ou região e, de alguma forma, buscar solucioná-los, ou seja, o ensino e a pesquisa devem ser direcionados para ações de impacto social que possibilitem melhores condições de vida para a população ${ }^{25}$. Diante dos resultados encontrados e o momento histórico da inserção da saúde bucal na ESF é necessária uma revisão da prática odontológica, principalmente nas 
ações de vigilância à saúde que envolve a promoção de saúde, prevenção de doenças e organização da demanda. O levantamento das informações sobre a percepção que o cirurgião-dentista tem quanto à participação dos agentes comunitário de saúde no desenvolvimento de ações vigilância à saúde bucal das famílias foi extrema importância na identificação de lacunas no modelo de atenção. Tendo em vista o papel de destaque dos ACS na vigilância à saúde bucal é necessário e oportuno, por meio de parceria UNIOESTE, $10^{\circ}$ Regional de Saúde do Paraná e Secretaria de Saúde de Cascavel, promover ciclos de debates e capacitação no sentido de aprimoramento da odontologia no SUS.

CONCLUSÃO

No contexto geral do estudo foi possível observar que a atuação dos cirurgiões-dentistas na capacitação continuada com a finalidade de proporcionar condições para uma atuação constante dos ACS no desenvolvimento das ações de saúde bucal pouco avançou, apesar de concordarem com a ideia da participação deste profissional nestas ações. O ACS pode atuar como agente transformador desta realidade, na medida em que está mais próximo da população, entretanto, é necessário qualificá-lo, para que este profissional possa exercer o papel que lhe cabe no âmbito da Odontologia. Mudanças no modelo de prestação dos serviços de saúde no município vão exigir a existência de um programa de educação permanente que possibilite renovar e adquirir os conhecimentos de saúde bucal para que os ACS consigam atuar como atores na vigilância em saúde bucal. Entretanto, para que isso se torne realidade, é imprescindível uma ação conjunta da Secretaria Municipal de Saúde, gerência da atenção básica, Equipe de Saúde da Família, ESB e do ACS.

\section{REFERÊNCIAS}

1. Brasil. Ministério da Saúde. Portaria $n^{\circ} 2.436$, de 21 de setembro de 2017, estabelece a revisão de diretrizes para a organização da Atenção Básica, no âmbito do SUS.

2. Carnut L, Filgueiras LV, Figueiredo N, Goes PSA. Validação inicial do índice de necessidade de atenção à saúde bucal para as equipes de saúde bucal na estratégia de saúde da família. Ciênc saúde coletiva. 2011; 16(7):3083-91.

3. Vettore MV, Marques RAA, Peres MA. Desigualdades sociais e doença periodontal no estudo SBBrasil 2010: abordagem multinível. Rev Saúde Pública. 2013; 47(suppl.3):29-39.

4. Barabato PR, Nagano, HCN, Zanchet FN, Boing $\mathrm{AF}$, Peres MA. Perdas dentárias e fatores sociais, demográficos e de serviços associados em adultos brasileiros: uma análise dos dados do Estudo Epidemiológico Nacional (Projeto SB Brasil 2002-2003). Cad Saúde Pública. 2007;
23(8):1803-14.

5. Moreira RS, Nico LS, Tomita NE. O risco espacial e fatores associados ao edentulismo em idosos em município do Sudeste do Brasil. Cad Saúde Pública. 2011; 27(10):2041-54.

6. Bornstein VJ, Stotz EN. Concepções que integram a formação e o processo de trabalho dos agentes comunitários de saúde: uma revisão da literatura. Ciênc saúde coletiva. 2008; 13(1):259-68.

7. Ferraz L, Aerts DRGC. O cotidiano de trabalho do agente comunitário de saúde no PSF em Porto Alegre. Ciênc saúde coletiva. 2005; 10(2):346-55.

8. Frazão P, Marques DSC. Influência de agentes comunitários de saúde na percepção de mulheres e mães sobre conhecimentos de saúde bucal. Ciênc saúde coletiva. 2006; 11(1):131-44.

9. Soares FF, Figueiredo CRV, Borges NCM, Jordão RA, Freire MCM. Atuação da equipe de saúde bucal na estratégia saúde da família: análise dos estudos publicados no período 20012008. Ciênc saúde coletiva. 2011; 16(7):316980.

10. Atlas Brasil 2013. Atlas do Desenvolvimento Humano no Brasil 2013. PNUD.

11. Morita MC, Haddad AE, Araújo ME. Perfil Atual e Tendências do Cirurgião-dentista Brasileiro. Maringá: Dental Press International; 2010.

12. Paranhos LR, Ricci ID, Scanavini MA, Bérzin F, Ramos AL. Análise do mercado de trabalho odontológico na região Sul do Brasil. RFO, Passo Fundo. 2009; 14(1):7-13.

13. Rodrigues AAAO, Santos AM, Assis MMA. Agente comunitário de saúde: sujeito da prática em saúde bucal em Alagoinhas, Bahia. Ciênc saúde coletiva. 2010; 15(3):907-15.

14. Campos L, Reiser LM, Bottan ER, Silveira EG. Conhecimento e atitudes sobre saúde bucal: estudo com agentes comunitários de saúde de Itajaí (SC). FOL • Faculdade de Odontologia de Lins/Unimep. 2015; 25(1):11-6.

15. Bortoli FR, Moreira MA, Kovaleski DF. Conhecimento dos Agentes Comunitários de Saúde em relação à saúde bucal. Sau \&Transf Soc. $2017 ;$ 8(2):96-102.

16. Mialhe FL, Lefèvre F, Lefèvre AMC. O agente comunitário de saúde e suas práticas educativas em saúde bucal: uma avaliação qualiquantitativa. Ciênc saúde coletiva. 2011; 16(11):4425-32.

17. Venancio EQ, Paula EMQV, Reis CB. Oral health care: the knowledge and work of the community health agent. Rev Odontol UNESP. 2014; 43(2):124-30.

18. Terreri ALM, Lima DP, Portinho D, Baltazar 
MMM, Berti M. Agentes Comunitários de Saúde: perspectivas, atividades preventivas e vigilância em saúde bucal da criança. Arch Health Invest. 2017; 6(5):225-30.

19. Moura MS, Carvalho CJ, Amorim JTC, Marques MFSS, Moura LFAD, Mendes RF. Perfil e práticas de saúde bucal do agente comunitário de saúde em municípios piauienses de pequeno porte. Ciênc saúde coletiva. 2010; 15(suppl.1):1487-95.

20. Freitas SFT, Calvo MCM, Lacerda JT. Saúde coletiva e novas diretrizes curriculares em Odontologia: uma proposta para graduação. Trab educ saúde. 2012; 10(2):223-34.

21. Brasil. Ministério da Saúde. Ministério da Educação. A aderência dos cursos de graduação em enfermagem, medicina e odontologia às diretrizes curriculares nacionais/ Ministério da Saúde, Ministério da Educação (Série F. Comunicação e Educação em Saúde). Brasília, DF: Ministério da Saúde, 2006. 162 p.

22. Kusma SZ, Moysés ST, Moysés SJ. Promoção da saúde: perspectivas avaliativas para a saúde bucal na atenção primária em saúde. Cad Saúde Pública. 2012; 28(suppl):s9-s19.

23. Brasil. Portaria $n^{\circ} 1.444$ de 28 de dezembro de 2000. Estabelece incentivo financeiro para a reorganização da atenção à saúde bucal prestada nos municípios por meio do Programa de Saúde da Família. Diário Oficial da União 2000.

24. Chaves SCL, Vieira-da-Silva LM. Atenção à saúde bucal e a descentralização da saúde no Brasil: estudo de dois casos exemplares no Estado da Bahia. Cad Saúde Pública. 2007; 23(5):1119-31.

25. Santos KT, Saliba NA, Moimaz SAS, Arcieri RM, Carvalho ML. Agente comunitário de saúde: perfil adequado a realidade do Programa Saúde da Família? Ciênc saúde coletiva. 2011; 16(supl.1):1023-28.

\section{CONFLITO DE INTERESSES}

Os autores declaram não haver conflitos de interesse.

\section{AUTOR PARA CORRESPONDENCIA}

\section{André Luiz Marçal Terreri}

andreterreri@hotmail.com

Submetido em 28/05/2018

Aceito em 06/06/2018 